Pensamiento Crítico N. ${ }^{\circ} 11$, pp. 11-32

\title{
¿Existe aún la industria manufacturera en el Perú?
}

\author{
Gilberto José Rafael Cárdenas Núñez
}

\section{RESUMEN}

La industria manufacturera peruana aún existe. Después de muchos años en que el proceso de liberalización de la economía sometió a las empresas industriales del sector a la competencia externa, se puede apreciar que más de 110 mil empresas existen y producen. Las principales son las que poseen 10 a más trabajadores, que producen más del $80 \%$ del total manufacturero nacional; se encuentran en Lima y Callao, o en las capitales de los departamentos, es industria ligera, poco articulada a los recursos naturales del país, de tecnología relativamente intermedia y que en gran parte del territorio nacional no existe.

Palabras clave: Industria, manufactura, producción, departamentos, economía.

\begin{abstract}
The manufacturing peruvian industry still exists. After many years in which the process of liberalization of the economy submitted to the industrial companies of the sector to the external competition(competence), it is possible to estimate that more than 110 thousand companies exist and produce. The principal ones are them of 10 to more workers, who produce more than $80 \%$ of the manufacturing national total, are principally in Lima and Callao, or in the capitales of the departments, it
\end{abstract}




\section{Gilberto José Rafael Cárdenas Núñez}

is a light(slight) industry little articulated to the natural resources of the country, of relatively intermediate technology and that largely of the national territory does not exist.

Keywords: Industry, manufacture, production, departments, economy.

\section{INTRODUCCIÓN}

La investigación y análisis del sector industrial manufacturero en el Perú es nuevamente un tema de primer orden. Desde hace muchos años no se publicaba información confiable sobre la estructura, el comportamiento o las características del sector manufacturero industrial peruano, razón por la cual los análisis al respecto fueron progresivamente desapareciendo de la literatura técnico económica y su número y calidad fueron disminuyendo hasta casi desaparecer.

Es gratificante para los investigadores de la realidad económica peruana que el Ministerio de la Producción esté desarrollando una importante iniciativa basándose en los resultados del Censo Nacional de Establecimientos Manufactureros, realizado en los años 2007 y 2008, “ . .. a fin de disponer de información confiable y actualizada sobre la actividad productiva del sector Industrial Manufacturero, que permitan determinar las características básicas de la estructura económica de las empresas y establecimientos por cada rubro del sector." 1

La importante iniciativa para conocer el sector industrial manufacturero está a cargo de un equipo técnico dirigido por J. Gambini M., quien labora como coordinador responsable de la actividad y está a la cabeza de un equipo de profesionales en este trabajo. $^{2}$

La crisis económica internacional de los años 2008-2009 originó que los profesionales y los estudiosos de los fenómenos económicos realizaran nuevas reflexiones acerca del rol del Estado respecto a las políticas económicas prevalecientes en muchos

1 Ministerio de la Producción; "Resultado por Regiones de la Actividad Económica de la Industria Manufacturera", p.7; Directorio Industrial, setiembre del 2009, Lima, Perú.

2 El equipo técnico está conformado por Gambini Martínez, Juan Feliz; Quispe Cacho, José; Oré Sánchez, Túrix Pedro y Figueroa Palomino, Renzo. 


\section{¿Existe aún la industria manufacturera en el Perú?}

países, tomando distancia de la aseveración que constituyó el paradigma predominante durante los últimos 20 años en el Perú. Tan concepción paradigmática, que proviene de las canteras del pensamiento neoclásico, se expresa radicalmente en el sentido que la mejor política industrial es la que no existe.

Tales ideas y concepciones ideológicas guían y guiaron a los investigadores cuyo tema central de estudio es la industria manufacturera en América Latina y el rol del Estado:

"En los años noventa, "protección", "sustitución de importaciones" e "intervención" se convirtieron en palabras malsonantes, en parte de la "leyenda negra" del desarrollo que experimentó la América Latina durante el periodo de posguerra. Como acontece con la mayoría de las "leyendas negras", ésta es a la vez verdadera y falsa"3.

\section{EMPRESAS Y ACTIVIDADES}

El Censo se realizó entre el 22 de octubre y el 31 de diciembre 2007. Para la ejecución del Censo se consideró como cobertura de realización 382 distritos urbanos a nivel nacional. Las variables que se tomaron en cuenta son: identificación y ubicación de empresa, razón social, actividad económica, inversiones, estado de pérdidas y ganancias, personal ocupado, cargas de personal ocupado, activo fijo, depreciación, insumos, materias primas, producción, ventas, consumo de energía, capacidad instalada, normas de gestión y de producto.

En una primera etapa, el registro de establecimientos manufactureros ubicados en 382 distritos urbanos permitió elaborar el directorio de las empresas manufactureras formales e informales (con o sin RUC). Luego, se aplicó la encuesta censal en profundidad, estratificada en empresas de 1 a 9 trabajadores por muestreo con encuestas personales, y a las empresas de 10 a más trabajadores por censo mediante la utilización de un cuestionario electrónico.

El importante trabajo ha dado como resultado la identificación y contabilización de 116,412 establecimientos manufactureros en todo el país, que conformaban 111,347

3 Cárdenas, Enrique; Ocampo, José Antonio y Thorp, Rosemary; "Industrialización y Estado en la América Latina”, p. 9; El Trimestre Económico, Fondo de Cultura económica, 2003, México. 


\section{Gilberto José Rafael Cárdenas Núñez}

empresas manufactureras. Esto es así porque una empresa puede tener más de un establecimiento, sea de producción, de almacenamiento u otro vinculado con la producción manufacturera.

La información publicada en el citado documento es muy importante y valiosa para que los investigadores se acerquen a la comprensión de las características de la inversión y producción industrial manufacturera peruana, las actividades en que se desarrolla la manufactura y otras características más. Así, en el primer cuadro que se presenta, los datos permiten identificar cinco primeras características del sector manufacturero industrial peruano.

Cuadro N. ${ }^{\circ}$ 1. Empresas por actividad industrial en la manufactura peruana.

\begin{tabular}{|c|c|c|c|}
\hline Orden de magnitud & Actividad industrial & Número de empresas & $\mathbf{\%}$ \\
\hline 1 & Textil & $23,391.00$ & 21.01 \\
\hline 2 & Madera y papeles & $19,269.00$ & 17.31 \\
\hline 3 & Metalmecánica & $16,671.00$ & 14.97 \\
\hline 4 & Agroindustria & $16,099.00$ & 14.46 \\
\hline 5 & Otras manufacturas & $14,080.00$ & 12.65 \\
\hline 6 & Edición e impresión & $9,250.00$ & 8.307 \\
\hline 7 & Pieles y cueros & $4,819.00$ & 4.328 \\
\hline 8 & Minería no metálica & $3,554.00$ & 3.192 \\
\hline 9 & Químicos & $2,602.00$ & 2.337 \\
\hline 10 & Joyas y artículos conexos & $1,114.00$ & 1 \\
\hline 11 & Pesca & 169.00 & 0.152 \\
\hline 12 & Siderometalúrgica & 156.00 & 0.14 \\
\hline 13 & Instrumentos de óptica y relojes & 136.00 & 0.122 \\
\hline 14 & Petróleo y derivados & 37.00 & 0.033 \\
\hline \multicolumn{2}{r|}{ Total manufactura } & $\mathbf{1 1 1 , 3 4 7 . 0 0}$ & $\mathbf{1 0 0}$ \\
\hline
\end{tabular}




\section{¿Existe aún la industria manufacturera en el Perú?}

Una primera aproximación a la comprensión de la información del Cuadro N ${ }^{\circ} 1$, permite señalar que el universo de empresas industriales manufactureras en el Perú es relativamente importante. Son 111,347 las empresas censadas en el 2007 y 2008.

La primera característica de la actividad industrial manufacturera en el Perú es su carácter de industria ligera, entendiendo como tal aquella cuya densidad de capital (capital fijo/trabajador) presenta un indicador relativamente bajo. Así, agregando a la industria textil la de madera y papeles, la agroindustria y otras manufacturas se aprecia que superan el $65 \%$ de las empresas existentes en el país. Sin embargo, con las actividades industriales de instrumentos de óptica y relojes, joyas y artículos conexos, pieles y cueros, edición e impresión, y otras, estamos hablando de la gran mayoría de industrias manufactureras peruanas.

La segunda característica que se puede apreciar es el casi insignificante desarrollo de la manufactura de bienes de capital. Realizando la agregación no depurada (puesto que en el número agregado se presentan también pequeñas empresas de industria ligera) de las actividades metalmecánica y la siderometalúrgica se alcanza al 15\% del universo de la manufactura peruana.

La tercera característica es la relativamente escasa manufactura de los principales recursos naturales nacionales, como son los minerales, el gas, petróleo y otros de menor importancia. La industria siderúrgica, la manufactura de productos de minería no metálica y la de petróleo y derivados comprenden a menos del $4 \%$ de empresas del país.

En el Gráfico N. ${ }^{\circ}$, se pueden distinguir claramente las órdenes de magnitud de las actividades manufactureras en el país. 


\section{Gilberto José Rafael Cárdenas Núñez}

\section{Grafico N. ${ }^{\circ} 1$}

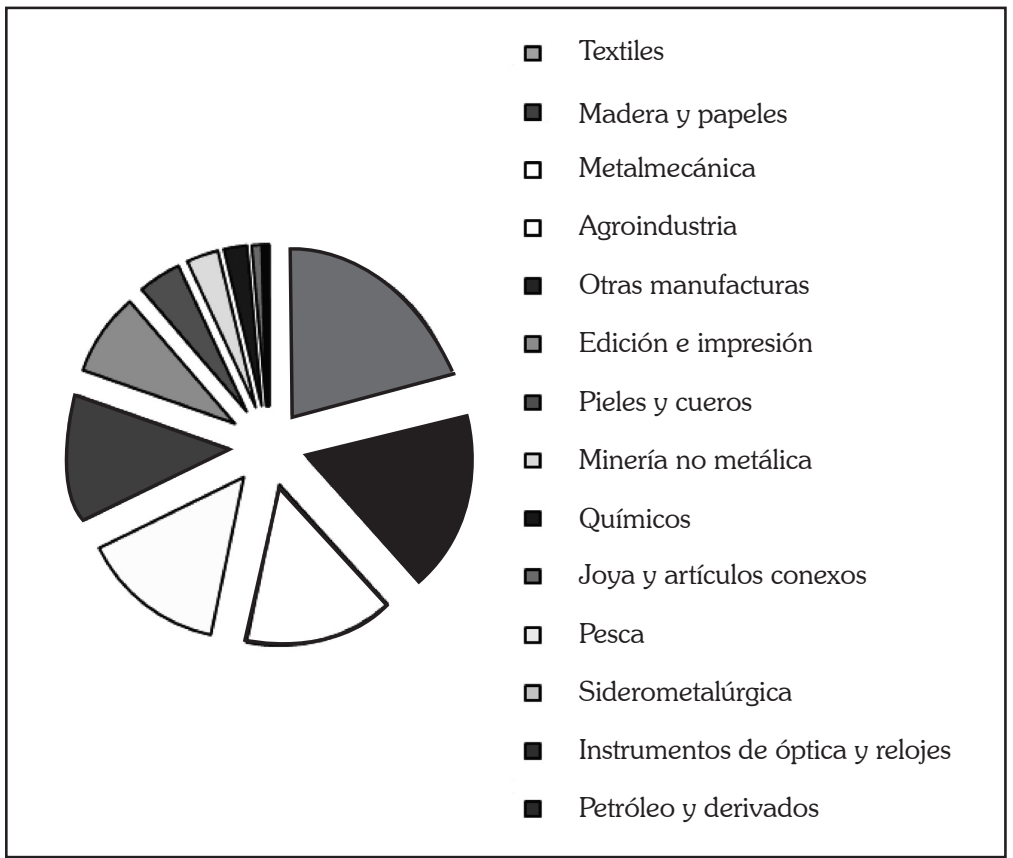

El área más grande representa a las industrias textiles, luego madera y papeles, metalmecánica, agroindustria y otras manufacturas. El resto de actividades cuenta con un número pequeño de empresas dedicadas a sus respectivas y limitadas producciones industriales.

\section{EMPRESAS MANUFACTURERAS POR DEPARTAMENTOS}

El directorio industrial también presenta información acerca de las empresas según su ubicación en el mapa político del país, es decir, según departamentos, aunque equívocamente las denomina regiones.

No es sorpresa descubrir que más del $50 \%$ de las empresas industriales manufactureras están en Lima Metropolitana. Y si al porcentaje de empresas ubicadas en Lima, se le añade el correspondiente a la Provincia Constitucional del Callao, se 
obtiene más del 55\% de las empresas ubicadas en el área de influencia de la capital política del país.

Cuadro N. ${ }^{\circ}$ 2. Empresas manufactureras por departamento.

\begin{tabular}{|c|c|c|c|}
\hline \multirow{2}{*}{$\begin{array}{l}\text { Orden de } \\
\text { magnitud }\end{array}$} & \multirow{2}{*}{ Departamento } & \multicolumn{2}{|c|}{ Empresas } \\
\hline & & Número & $\%$ \\
\hline 1 & Lima & 58,475 & 52.52 \\
\hline 2 & Arequipa & 6,942 & 6.235 \\
\hline 3 & La Libertad & 4,966 & 4.46 \\
\hline 4 & Junín & 4,888 & 4.39 \\
\hline 5 & Puno & 3,458 & 3.106 \\
\hline 6 & Piura & 3,408 & 3.061 \\
\hline 7 & Cusco & 3,243 & 2.912 \\
\hline 8 & Callao & 3,180 & 2.856 \\
\hline 9 & Lambayeque & 3,077 & 2.763 \\
\hline 10 & Ancash & 2,672 & 2.4 \\
\hline 11 & Tacna & 2,616 & 2.349 \\
\hline 12 & San Martín & 2,129 & 1.912 \\
\hline 13 & Cajamarca & 1,996 & 1.793 \\
\hline 14 & Ica & 1,660 & 1.491 \\
\hline 15 & Loreto & 1,390 & 1.248 \\
\hline 16 & Ayacucho & 1,189 & 1.068 \\
\hline 17 & Huánuco & 1,126 & 1.011 \\
\hline 18 & Ucayali & 1,078 & 0.968 \\
\hline 19 & Moquegua & 994 & 0.893 \\
\hline 20 & Apurímac & 768 & 0.69 \\
\hline 21 & Pasco & 596 & 0.535 \\
\hline 22 & Amazonas & 542 & 0.487 \\
\hline 23 & Tumbes & 382 & 0.343 \\
\hline 24 & Huancavelica & 362 & 0.325 \\
\hline 25 & Madre de Dios & 211 & 0.189 \\
\hline
\end{tabular}




\section{Gilberto José Rafael Cárdenas Núñez}

Una cuarta característica de la industria manufacturera en el Perú es que además de centralizada en la capital de la República, la mayoría de las empresas está en las ciudades capitales de departamento cercanas al litoral. Es así que Arequipa, con sus puertos de Mollendo y Matarani, vinculados a la ciudad mediante carreteras de buena calidad y ferrocarril para carga pesada, se suma a La Libertad que también cuenta con facilidades de comercio hacia el exterior, y Junín que se vincula con Lima y la Provincia Constitucional del Callao mediante el ferrocarril central y la Carretera Central.

La quinta característica notable es la casi inexistencia de industria manufacturera por lo menos en ocho departamentos del país que, salvo Tumbes, están en la sierra y selva, y muy alejados del litoral.

La configuración del mapa manufacturero del Perú es de una costa con industria manufacturera relativamente importante, con ciudades capitales de departamento del interior relativamente industrializadas con manufactura, vinculadas al litoral mediante vías de buena o regular calidad (Puno, Cusco), y por último una sierra y selva extractivas o productoras de alimentos $\mathrm{u}$ otros sin mayor grado de transformación ni valor agregado.

Gráfico N. ${ }^{\circ} 2$

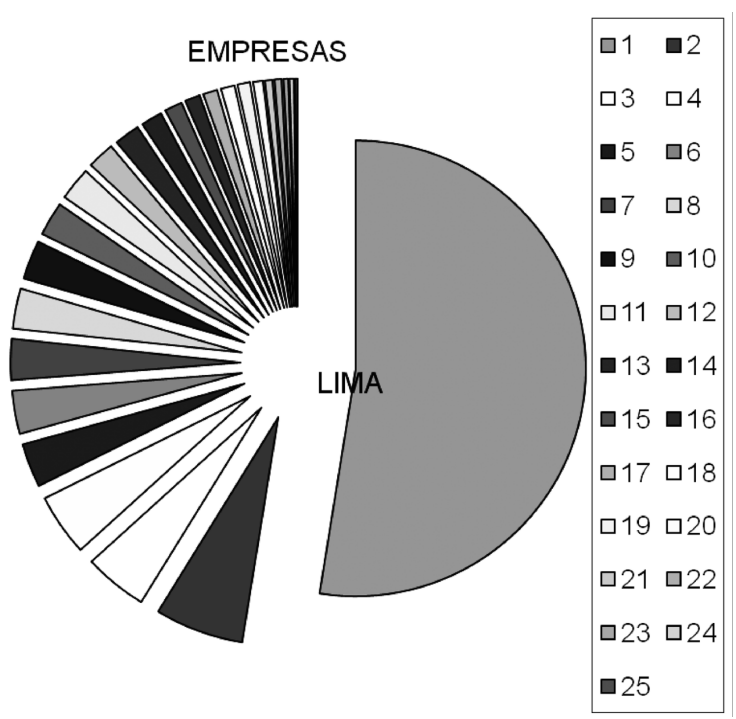




\section{¿Existe aún la industria manufacturera en el Perú?}

En el Gráfico N. ${ }^{\circ} 2$ se puede apreciar la presencia dominante y manufacturera de Lima Metropolitana como centro de la industria manufacturera peruana.

Las razones de tal concentración son las siguientes:

1. La infraestructura productiva confiable para una producción sostenible y que pueda ser competitiva se encuentra en Lima Metropolitana y en la Provincia Constitucional del Callao. Las condiciones de infraestructura se refieren a los servicios de abastecimiento de energía eléctrica, de agua potable y alcantarillado, y el acceso al comercio exterior.

2. La maestranza disponible en la capital permite a los empresarios la superación o arreglo de fallas y desperfectos en la maquinaria en forma rápida, oportuna y relativamente económica.

3. La cercanía del puerto, aeropuerto y otros medios de comunicación.

4. La posibilidad de conseguir el concurso de mano de obra especializada, ingeniería y gerencia.

\section{LAS PRINCIPALES EMPRESAS INDUSTRIALES DEL PAÍS}

Teniendo en consideración algunas limitaciones propias de la información disponible, como que la información de las empresas en Lima Metropolitana es hasta el número de 400 trabajadores, mientras la de los otros departamentos es de hasta 100 trabajadores, a continuación se presentan las principales empresas manufactureras del Perú según el número de trabajadores. 


\section{Gilberto José Rafael Cárdenas NúñeZ}

Cuadro N. ${ }^{\circ}$ 3. Principales empresas manufactureras más grandes del país.

\begin{tabular}{|c|c|c|c|c|}
\hline Ord. & Razón social & Actividad económica & Trab. & Ubicación \\
\hline 1 & Emp. Ag. Casagrande & Elab. Azúcar & 4000 & La Libertad \\
\hline 2 & SPCC & Fab. Prod. Prim. Metales & 3839 & Lima \\
\hline 3 & Confecciones Textimax S.A. & Conf. Prend. Vestir Tej. Punto & 3371 & Lima \\
\hline 4 & Emp. Ag. Pomalca S.A. & Elaboración de Azúcar & 3236 & Lambayeque \\
\hline 5 & Emp. Madedera Sullana S.A. & Const. Mód. Pre Fabricados & 3000 & Lima \\
\hline 6 & Emp. Ag. Tumán S.A. & Elab. Azúcar Rubia y Refinada & 2947 & Lambayeque \\
\hline 7 & Petróleos del Perú Petroperú & Elaboración de Combustibles & 2752 & Lima \\
\hline 8 & Diseño y Color S.A. & Fab. Polos y Ropa de Vestir & 2343 & Lima \\
\hline 9 & Textil San Cristóbal S.A. & Fab. Prendas Vestir Tej. Punto & 2240 & Ica \\
\hline 10 & Austral Gruop S.A. & Elab. Harina y Conservas & 2000 & Lima \\
\hline 11 & Sociedad Agrícola Drokasa S.A. & Procesamiento de Espárragos & 2000 & Ica \\
\hline 12 & Empresa Siderúrgica del Perú & Fab. Productos Siderúrgicos & 1925 & Ancash \\
\hline 13 & Nestlé Perú S.A. & Elaboración de Helados & 1801 & Lima \\
\hline 14 & Serv. Ind.de La Marina & Fab. de Estructuras Metálicas & 1658 & Callao \\
\hline 15 & Cottol Bel Eirl & Conf. de Prendas de Vestir & 1600 & Lima \\
\hline 16 & Texfina S.A. & Fabricación de Telas & 1373 & Ica \\
\hline 17 & Corp. Fabril Confecciones S.A. & Fab. de Polos y Telas & 136 & Lima \\
\hline 18 & Complejo Ag. Cartavio S.A.C. & Elab. Azúcar, Alcohol y Melaza & 1340 & La Libertad \\
\hline 19 & Agroindustrial Paramonga & Elaboración de Azúcar & 1250 & Lima \\
\hline 20 & Corp. Texpop S.A. & Fab. de Prendas de Vestir & 1236 & Lima \\
\hline 21 & Cementos Lima S.A. & Fab. Envas. de Cementos & 1200 & Lima \\
\hline 22 & Compañía Universal Textil S.A. & Fab. Fibras Textiles & 1194 & Lima \\
\hline 23 & Gloria S.A. & Adm. Elab. de Leche & 1175 & Lima \\
\hline 24 & Co.Ind. Text. CredisaTrutex S.A.C. & Fab. de Hilos y Telas & 1171 & Lima \\
\hline 25 & Industrias Nettalco S.A. & Fabrica Textil & 1143 & Lima \\
\hline 26 & Ian Perú S.A.C. & Conservación de Frutas & 1118 & Lima \\
\hline 27 & Topy Top S.A. & Fab. Telas y Polos & 1005 & Lima \\
\hline 28 & Samitex S.A. & Fab. Telas & 1000 & Lima \\
\hline 29 & Intitex S.A. & Conf. de Prendas de Vestir & 1000 & Lima \\
\hline 30 & Pez Mundo Int. Corp. S.A. & Proc. de Anguila Congelada & 901 & Piura \\
\hline
\end{tabular}




\section{¿Existe aún la industria manufacturera en el Perú?}

\begin{tabular}{|c|c|c|c|c|}
\hline Ord. & Razón social & Actividad económica & Trab. & Ubicación \\
\hline 31 & Cotton Knit S.A.C. & Fab. Prendas de Vestir & 968 & Lima \\
\hline 32 & Cia Ind. Nuevo Mundo S.A. & Fabricación de Telas & 954 & Lima \\
\hline 33 & Kraft Foods Perú S.A. & Fab. Confitería y Galletas & 946 & Lima \\
\hline 34 & Yobel Sup. Chain Management & Fab. Productos de Limpieza & 946 & Lima \\
\hline 35 & Pesquera Exalmar S.A. & Conservación de Pescado & 903 & Lima \\
\hline 36 & Ind.Pesquería Sta Mónica S.A. & Proc. Prod Hidrobiológicos & 881 & Piura \\
\hline 37 & Perú Fashions S.A.C. & Fab. Prendas Vestir & 850 & Lima \\
\hline 38 & Danper Trujillo S.A.C. & Fab. Espárr. Hort.Legumbres & 844 & La Libertad \\
\hline 39 & Corp. Grafica Navarrete S.A. & Actividades de Impresión & 813 & Lima \\
\hline 40 & Emp. Ag. Laredo S.A.C. & Elaboración de Azúcar & 809 & La Libertad \\
\hline 41 & Hiland. Algodón Peruano S.A. & Hilandería & 800 & Lima \\
\hline 42 & Unión de Concreteras S.A. & Prep Mezcla de Concreto & 800 & Lima \\
\hline 43 & Corp. José R. Lindley S.A. & Elab. Bebidas Gaseosas & 792 & Lima \\
\hline 44 & Emp Periodística Nacional S.A. & Impresión Diarios Ojo y Correo & 744 & Lima \\
\hline 45 & Emp.Editora El Comercio S.A. & Edición y Diseño Periódicos & 735 & Lima \\
\hline 46 & Industrias Teal S.A. & Elaboración de Golosinas & 726 & Lima \\
\hline 47 & Calzado Chosica S.A. Cerrada & Fab. Calzado Maquillador & 700 & Lima \\
\hline 48 & Armador. Cong. del Pacífico S.A. & Proc. Congelado y Exportación & 700 & Piura \\
\hline 49 & Pesquera Hayduk S.A. & Elab. Productos de Pescado & 677 & Lima \\
\hline 50 & Texgroup S.A. & Fab. Prendas de Vestir & 660 & Lima \\
\hline 51 & Modas Diversas del Perú & Conf.de Prendas de Vestir & 635 & Lima \\
\hline 52 & Indelat S.A.C. & Fab. Planchas Microporosas & 625 & Lima \\
\hline 53 & Dino S.A.C. & Fabricación de Cemento & 607 & La Libertad \\
\hline 54 & Twilltex S.A. & Fab. Prendas de Vestir & 606 & Lima \\
\hline 55 & Carapez Traders S.A.C. & Proc de Prod. Hidrobiológicos & 601 & Piura \\
\hline 56 & Agropucalá S.A.C. & Elaboración de Azúcar & 600 & Lambayeque \\
\hline 57 & Charpas S.R.L. & Fabricación de Joyas & 593 & Lima \\
\hline 58 & Productos Paraiso del Perú & Fabricación de Colchones & 580 & Callao \\
\hline 59 & El Portillo S.R.L. & Fab. Estructuras Metálicas & 564 & Moquegua \\
\hline 60 & Ref. Zinc Cajamarquilla, S.A. & Elab. Refinado Electrolítico & 550 & Lima \\
\hline 61 & Hersil S.A. Lab. Ind. Farmacéut. & Fab. Línea Farmacéutica & 539 & Lima \\
\hline 62 & Man. Pap. y Cartones Perú S.A. & Depósito de la Fábrica. & 532 & Lima \\
\hline
\end{tabular}




\section{Gilberto José Rafael Cárdenas NúñeZ}

\begin{tabular}{|c|c|c|c|c|}
\hline Ord. & Razón social & Actividad económica & Trab. & Ubicación \\
\hline 63 & Ajinomoto del Perú S.A. & Elab. de Alim.Condimentos & 530 & Lima \\
\hline 64 & Ajeper S.A. & Elab. Bebidas Gaseosas & 520 & Lima \\
\hline 65 & Braedt S.A. & Fabricación de Embutidos & 500 & Lima \\
\hline 66 & Industrias del Bordado S.A. & Industria del Bordado & 500 & Lima \\
\hline 67 & Ransa Comercial S.A. & Elab. Cons. Prod Pescado & 500 & Callao \\
\hline 68 & Tal S.A. & Elab. de Cons. Espárrago & 500 & La Libertad \\
\hline 69 & Ferreyros S.A.C. & Obras Ingeniería Mecánica & 490 & Lima \\
\hline 70 & Pesquera Ind. El Angel S.A. & Elab. y Conserv.de Pescado & 485 & Lima \\
\hline 71 & Arin S.A. & Fab. Prod. Prim.Met. Preciosos & 485 & Lima \\
\hline 72 & Corp. Infamasa S.A. & Laboratorio Farmacéutico & 464 & Lima \\
\hline 73 & Ucp Backus y Johnson S.A.C. & Fabricación de Cerveza & 463 & Arequipa \\
\hline 74 & Industrial Ucayali S.A.C. & Aserradero y Transf. Madera & 450 & Ucayali \\
\hline 75 & Pesquera Diamante S.A. & Oficina Industria Pesquera & 420 & Callao \\
\hline 76 & Negociación Lanera del Perú & Fab. Hil. Lana, Fibra Acrílica & 417 & Callao \\
\hline 77 & Industrias Electroquímicas S.A. & Fabricación de Insecticidas & 410 & Callao \\
\hline 78 & Seafrost S.A. & Proc. y Congelado Pescado & 408 & Piura \\
\hline 79 & Fundición Callao S.A. & Fund.de Metal de Todo Tipo & 400 & Callao \\
\hline 80 & Procesadora S.A.C. & Elab. Frut Legumb Hortalizas & 351 & Lambayeque \\
\hline 81 & Inversiones Himalaya S.A. & Prod. de Cons.y Semicons. & 350 & Ica \\
\hline 82 & Procesadora Laran S.A.C. & Procesadora de Cítricos & 350 & Ica \\
\hline 83 & Bsh Electrodomésticos S.A.C. & Fab. Com. Electrodom L. Blan. & 337 & Callao \\
\hline 84 & Cerámica Lima S.A. & Depósito & 333 & Callao \\
\hline 85 & Panificadora Bimbo Perú S.A. & Elaboración Panes & 327 & Callao \\
\hline 86 & Industrias Vencedor & Depósito de Pintura Vencedor & 322 & Arequipa \\
\hline 87 & Agroindustrias San Jacinto S.A.C. & Elab. de Azúcar y Alcohol & 310 & Ancash \\
\hline 88 & Freno S.A. & Fab. Autopartes para Carros & 306 & Callao \\
\hline 89 & Espárragos del Sur S.A.C. & Procesamiento de Espárragos & 300 & Ica \\
\hline 90 & Sociedad Agrícola Drokasa S.A. & Export.Espár. Uva y Palta & 300 & Ica \\
\hline 91 & La Colonial Fabrica de Hilos & Fabricación de Hilos & 300 & Callao \\
\hline 92 & Propac & Fab. Mallas Eléctricas & 300 & Callao \\
\hline 93 & Triplay Amazónico S.A.C. & Producción de Triplay & 290 & Ucayali \\
\hline 94 & Cemento Sur S.A. & Fab. Cemento Portland Cal Hid & 280 & Puno \\
\hline
\end{tabular}




\section{¿Existe aún la industria manufacturera en el Perú?}

\begin{tabular}{|c|c|c|c|c|}
\hline Ord. & Razón social & Actividad económica & Trab. & Ubicación \\
\hline 95 & Ind. Chucarapi Pampa Blanca & Proc. Caña Azúcar y Deriv. & 278 & Arequipa \\
\hline 96 & Alimentos Procesados S.A.C. & Elab. Alimentos Procesados & 270 & Arequipa \\
\hline 97 & Textiles Del Sur S.A.C. & Elab. Fibras Hilo Tejeduría & 268 & Ica \\
\hline 98 & Manufacturas del Sur S.A. & Elab. Fibras Sintéticas & 262 & Arequipa \\
\hline 99 & Yura S.A. & Fab. Cemento, Cal y Yeso & 250 & Arequipa \\
\hline 100 & Quimpac S.A. & Fab. Productos Químicos & 250 & Callao \\
\hline 101 & Cía Gooyear del Perú S.A. & Fab. Llantas de Caucho & 250 & Callao \\
\hline 102 & Corporación Cerámica S.A. & Fab. Sanitarios Mayólica & 250 & Callao \\
\hline 103 & El Pedregal S.A. & Cultivo, Empaque y Exp Uva & 250 & Ica \\
\hline 104 & Corp. Ingeniería Refrig. S.R.L. & Elab. Cong. Prod. Hidrobiológicos & 250 & Piura \\
\hline 105 & Sakana del Perú S.A. & Proc. Exp. Productos Marinos & 245 & Piura \\
\hline 106 & Industrias del Envase S.A. & Fab. Envases de Plástico & 242 & Callao \\
\hline 107 & Cemento Andino S.A. & Fabricación de Cemento & 237 & Junín \\
\hline 108 & Cfg Investtment S.A.C. & Harina Conservas de Pescado & 230 & Ancash \\
\hline 109 & Corporación Cervesur S.A.C. & Fabricación de Cerveza & 230 & Arequipa \\
\hline 110 & Agroindustrias San Jacinto S.A.C. & Oficinas Administrativas & 220 & Ancash \\
\hline 111 & Cervecera San Juan S.A.C. & Elabor. de Bebidas Heladas & 209 & Ucayali \\
\hline 112 & Corporación Rey S.A. & Fab. Cierres de Cremallera & 201 & Callao \\
\hline 113 & Consorcio Perú Murcia S.A.C. & Prod. y Empac. de Alcachofas & 200 & Arequipa \\
\hline 114 & Productos del Sur S.A. & Producción de Textiles & 200 & Arequipa \\
\hline 115 & Molino del Triunfo S.A. & Fideos de Harina Deriv. Trigo & 200 & Callao \\
\hline 116 & Haug S.A. & Fab. Fierros para Construcción & 200 & Callao \\
\hline 117 & Peak Quality del Perú S.A. & Procesamiento de Espárragos & 200 & Ica \\
\hline 118 & Grupo Gloria S.A.C. & Fabricación de Papel y Cartón & 200 & La Libertad \\
\hline 119 & Corp. Aceros Arequipa S.A. & Laminación de Acero Caliente & 196 & Arequipa \\
\hline 120 & Desarrollo Forestal & Aserrío y Cepillado de Madera & 189 & Loreto \\
\hline 121 & Triplay Enchapes S.A.C. & Fabricación de Triplay & 182 & Loreto \\
\hline 122 & Génesis Eirl & Fab. Conservas de Pescado & 180 & Ancash \\
\hline 123 & Cons. Derivad San Andrés S.A.C. & Fab. Cons. y Filetes Anchoas & 180 & Ica \\
\hline 124 & Tableros Peruanos & Fab. de Hojas de Madera & 180 & La Libertad \\
\hline 125 & Peruana de moldeados S.A. & Fab. Plasticos Bolsas y Deriv & 179 & Callao \\
\hline 126 & Owens Illinois Perú S.A. & Prendas de Vestir & 177 & Callao \\
\hline
\end{tabular}




\section{Gilberto José Rafael Cárdenas NúñeZ}

\begin{tabular}{|c|c|c|c|c|}
\hline Ord. & Razón social & Actividad económica & Trab. & Ubicación \\
\hline 127 & Triplay Martín S.A.C. & Fabricación de Triplay & 173 & Loreto \\
\hline 128 & Distribuciones de Frio S.A.C. & Venta de Helados & 170 & Arequipa \\
\hline 129 & Maquimar S.A. & Fábrica de Conservas & 165 & Callao \\
\hline 130 & Anchoveta S.A. & Transf. Prod. Hidrobiológicos & 160 & Ica \\
\hline 131 & Molinera Inca S.A. & Venta de Galletas & 160 & La Libertad \\
\hline 132 & Firma S.A. & Fab. Estructuras Metálicas & 158 & Callao \\
\hline 133 & Inkabor S.A.C. & Transformación de Boro & 155 & Arequipa \\
\hline 134 & Prexair Perú S.A. & Fab. Sust. Químicas Básicas & 154 & Callao \\
\hline 135 & Vásquez Cáceres, José & Distribuidora de Gaseosas & 151 & Cusco \\
\hline 136 & Neg. Maderera Travis S.R.L. & Maderera y Aserraderos & 151 & Junín \\
\hline 137 & Santa Cruz Inversiones S.A.C. & Fab. Conservas de Pescado & 150 & Ancash \\
\hline 138 & Agroinca Prod. Per. de Export & Fab. Productos Lácteos & 150 & Arequipa \\
\hline 139 & Internacional de Comercio S.A. & Fab. Textilera, Hilos Fibras & 150 & Arequipa \\
\hline 140 & Cons. Ind. de Arequipa S.A. & Fab. Jabón Tocador. Vaselina & 150 & Arequipa \\
\hline 141 & Embotelladora Frontera S.A. & Depósito & 150 & Arequipa \\
\hline 142 & Fábrica de Cintas Arbona S.A. & Fab. Cintas de Pasamanería & 150 & Callao \\
\hline 143 & Pesquera Hayduk S.A. & Elab. Cons. Cong. Pescado & 150 & Moquegua \\
\hline 144 & Funsur S.A. & Fundic. y Refinación de Estaño & 148 & Ica \\
\hline 145 & Envases Industriales & Fab. Env. Plástico y Cartón & 146 & Callao \\
\hline 146 & Emp. Ed. La Industria Trujillo S.A. & Ed., Impresión Venta Diarios & 145 & La Libertad \\
\hline 147 & Talara Catering Service S.A.C. & Elaborac Bocaditos Pasteles & 145 & Piura \\
\hline 148 & Franky y Ricky S.A. & Fab. Prend. Algod. Tejido Punto & 140 & Arequipa \\
\hline 149 & Ind. Pesquera y Servicios S.A. & Elab. Cons. Prod Pescado & 140 & Callao \\
\hline 150 & Sulfato de Cobre S.A. & Elab. Productos Químicos & 135 & Callao \\
\hline 151 & Sudamericana de Fibras S.A. & Industria de Fibras Acrílicas & 133 & Callao \\
\hline 152 & Inversiones Regal & Fab. Conservas de Pescado & 130 & Ancash \\
\hline 153 & Fábrica de Choc. La Ibérica S.A. & Fab. Chocolates y Confituras & 130 & Arequipa \\
\hline 154 & Productos Forjados S.A.C. & Fab. de Accesorios de Minería & 130 & Callao \\
\hline 155 & Conservera Garrido S.A. & Cons. Env. Pescado Fresco & 130 & Piura \\
\hline 156 & Freeko Perú S.A. & Proces. Enlat. Prod. Hidrobiol & 128 & Piura \\
\hline 157 & Moly-Cop Perú S.A.C. & Elaboración de Alimentos & 125 & Arequipa \\
\hline 158 & Italmecan S.A. & Fab. Piezas Metál. Emb. Pesq. & 123 & Callao \\
\hline
\end{tabular}




\section{¿̇existe aún la industria manufacturera en el Perú?}

\begin{tabular}{|l|l|l|l|l|}
\hline Ord. & \multicolumn{1}{|c|}{ Razón social } & \multicolumn{1}{|c|}{ Actividad económica } & Trab. & \multicolumn{1}{|c|}{ Ubicación } \\
\hline 159 & Coop. Ind. Manuf Centro Ltda & Fab. Paños Telas Calad. Lana & 123 & Junín \\
\hline 160 & Granja Rinconada del Sur S.A. & Planta de Beneficio de Aves & 122 & Arequipa \\
\hline 161 & Actividades Pesqueras S.A. & Harina y Cons de Pescado & 120 & Ancash \\
\hline 162 & Productores Agríc. Siguas S.A.C. & Prod. Agríc. de Exportación & 120 & Arequipa \\
\hline 163 & Ladrillera El Diamante S.A.C. & Elab. de Ladrillos y Afines & 120 & Arequipa \\
\hline 164 & Alusud Perú S.A. & Fab. Tapas Roscas Gaseosas & 120 & Callao \\
\hline 165 & Colgate Palmolive Perú S.A. & Almacen de Prod.de Higiene & 120 & Callao \\
\hline 166 & Empac. y Proc. Huamaní S.A.C. & Proc. y Export Espárragos & 120 & Ica \\
\hline 167 & Sami Iquitos S.R.L. & Const. de Barcos y Lanchas & 120 & Loreto \\
\hline 168 & Perú Timber S.A.C. & Aserrío de Madera & 120 & Ucayali \\
\hline 169 & Mad. Ind. y Laminadas S.A.C. & Fabricac. de Hojas de Triplay & 119 & Ucayali \\
\hline 170 & Ind. Molinera Rojas Eirl & Mol. Arroz & 118 & San Martín \\
\hline 171 & Milanovich Nieto Carlos & Cong de Prod. Hidrobiológicos & 115 & Piura \\
\hline 172 & Pesquera Diamante S.A. & Industria Pesquera & 110 & Callao \\
\hline 173 & Forestal Industrial Yaraví S.A. & Aserrío y Trat. Químico Madera & 110 & Loreto \\
\hline 174 & Papelera Panamericana S.A. & Fábrica de Papeles & 109 & Arequipa \\
\hline 175 & Agricultores Productores & Procesamiento de Espárragos & 109 & Ica \\
\hline 176 & Pesquera Diamante S.A. & Harina Conservas de Pescado & 106 & Ancash \\
\hline 177 & Cementos Selva S.A. & Fab. Cemento, Cal y Yeso & 106 & San Martín \\
\hline 178 & Postes del Altiplano S.A. & Fab. Postes de concreto & 104 & Puno \\
\hline 179 & Basf Peruana S.A. & Fab. Otros Pdts. Químicos Ncp & 103 & Callao \\
\hline
\end{tabular}

El criterio del número de trabajadores para el orden de tamaño no es siempre exacto, pues existen otros criterios como el del capital invertido, la densidad de capital, el valor agregado bruto o el valor bruto de producción. Si se toma en cuenta el criterio de tamaño según la cantidad de trabajadores declarados por la empresa, las principales empresas industriales manufactureras peruanas serían azúcareras o textiles, pues 3 de las 10 primeras empresas se dedican a la actividad agroindustrial azúcarera, o sea, los llamados ingenios azúcareros, o bien a la producción de prendas de vestir.

Solo una empresa minera aparece entre las manufactureras principales por el número de trabajadores: la Southern Perú Cooper Corporation. En realidad lo que sig- 


\section{Gilberto José Rafael Cárdenas Núñez}

nifican las cifras del cuadro $\mathrm{N}^{\circ} 3$, es que las actividades industriales manufactureras intensivas en mano de obra son esas, las agroindustrias y las textiles.

Por ello es que si se organiza la información de otra manera, por ejemplo por el valor total de la producción de la empresa en un año, el orden de tamaño varía sustancialmente, pues algunas empresas que tienen relativamente menos trabajadores pueden tener mayor nivel de ventas por el valor del producto que generan. Es así que PETROPERÚ, con las ventas que realiza en el mercado interno puede hacer que la actividad de producción industrial de coque y refinados de petróleo ocupe una posición relevante, así como la empresa específicamente mencionada.

Situación similar se presenta en las actividades de producción industrial de bebidas, específicamente con las de cerveza, en la actividad de hierro, acero y no ferrosos, en la cual dos empresas principales hacen prácticamente toda la actividad manufacturera nacional (SIDERPERÚ y Aceros Arequipa) ambas con altos niveles de densidad de capital pero con relativamente menos trabajadores.

La información presentada por el Censo Nacional Manufacturero tiene alto grado de confiabilidad, aproximándose al $100 \%$ de los resultados reales de la actividad productiva, y registrando en el campo datos otorgados por las empresas censadas. 
¿Existe aún la industria manufacturera en el Perú?

Cuadro N. ${ }^{\circ}$ 4. Empleo en las actividades industriales manufactureras (millones de nuevos soles)

\begin{tabular}{|l|r|}
\hline \multicolumn{1}{|c|}{ División CIIU } & Estimado \\
\hline Total & $\mathbf{7 4 8 , 6 3 6}$ \\
\hline 15 Alimentos y bebidas & 215,237 \\
\hline 16 Tabaco & 109 \\
\hline 17 Productos textiles & 62,365 \\
\hline 18 Prendas de vestir y art. piel & 113,241 \\
\hline 19 Cuero y calzado cuero y plast. & 26,318 \\
\hline 20 Madera y prods. madera & 20,827 \\
\hline 21 Papel, libros y otros & 6,330 \\
\hline 22 Periódicos e impresos & 45,977 \\
\hline 23 Coque y refin. petróleo & 3,561 \\
\hline 24 Químicos y derivados & 31,130 \\
\hline 25 Caucho y plásticos & 24,530 \\
\hline 26 Vidrio, cemento, cerámicos y ot. & 25,385 \\
\hline 27 Hierro, acero y no ferrosos & 14,447 \\
\hline 28 Productos metálicos & 56,431 \\
\hline 29 Maquinarias, armas y otros & 15,163 \\
\hline 30 Otros & 653 \\
\hline 31 Motores y equipos eléctricos & 5,857 \\
\hline 32 Productos de radio y TV. & 249 \\
\hline 33 Equipo médico e inst. medición & 2,608 \\
\hline 34 Automotores, partes y piezas & 6,816 \\
\hline 35 Equipo de transporte & 4,035 \\
\hline 36 Muebles, joyas y prod. juegos & 65,246 \\
\hline 37 Reciclamiento met. y no met. & 2,122 \\
\hline & \\
\hline & \\
\hline
\end{tabular}

El personal ocupado en el sector manufacturero de 10 a más trabajadores es relativamente menor al personal ocupado en el sector de 9 a menos trabajadores, pero la 


\section{Gilberto José Rafael Cárdenas Núñez}

actividad permanente de las empresas industriales manufactureras (entre 11 y 12 meses al año de actividad), garantiza el flujo de valor agregado hacia la economía (remuneraciones, utilidades, rentas, etc.) ejerciendo un efecto importante en la demanda agregada.

Gráfico N. ${ }^{\circ}$ 1. Personal ocupado por CIIU del Censo de Empresas Manufactureras, según división CIIU 2007.

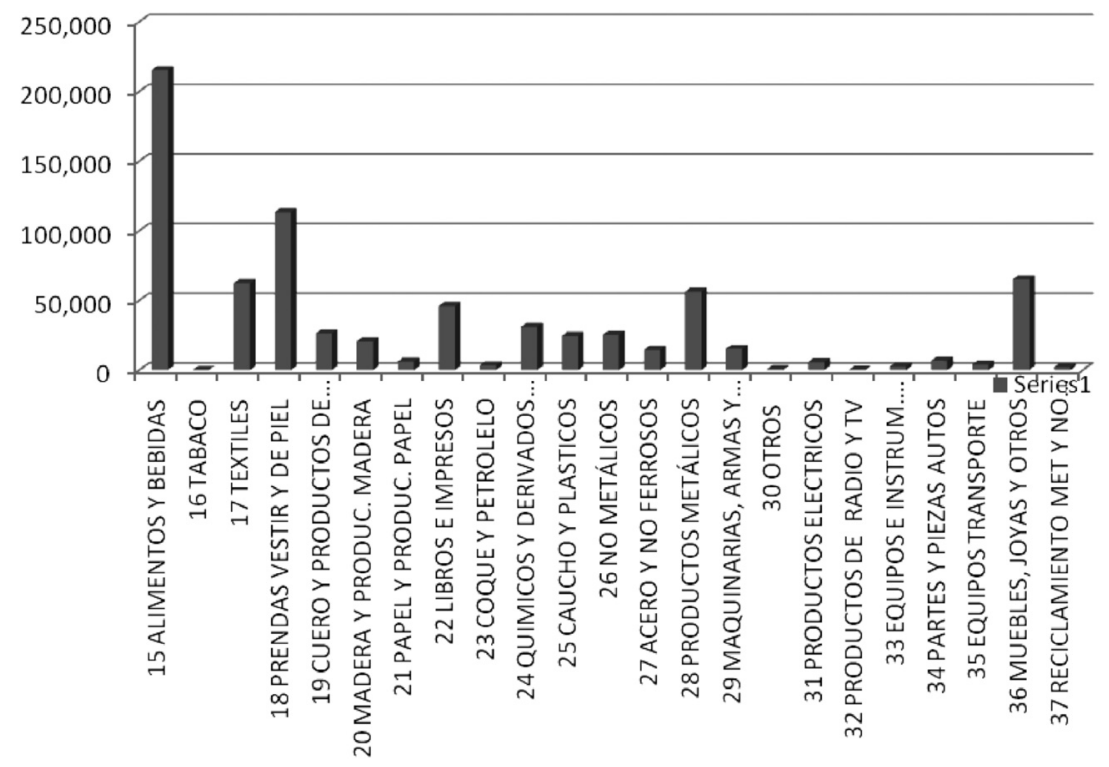

La actividad de alimentos y bebidas es la más importante de la actividad manufacturera en cuanto a creación de empleo permanente y formación de la masa de capacidad adquisitiva por el lado de la demanda agregada. Le siguen las actividades de producción de prendas de vestir y de piel, y de muebles, joyas y otros en ese orden. También son importantes actividades generadoras de empleo las actividades textiles y de fabricación de productos metálicos.

Por otro lado, generan muy poco empleo las actividades de refinación de petróleo, tabaco, productos de radio y televisión, equipos e instrumentos de medición, equipos de transporte y las actividades de reciclamiento de metálicos y no metálicos. 


\section{¿Existe aún la industria manufacturera en el Perú?}

Sin embargo, los empleos que generan las actividades más importante desde el punto de vista del empleo carecen de nivel de desarrollo tecnológico contemporáneo, pues muchas son panaderías y pastelerías, fábricas textiles y de prendas de vestir intensivas en mano de obra, y las empresas de transformación de la madera.

A la inversa es el caso de la actividad de refinería de petróleo y la fabricación de productos de tabaco en donde la tecnología es más importante, aunque también generan externalidades negativas propias del fenómeno de los desechos y de los desperdicios de alto riesgo ecológico.

Con las aclaraciones realizadas se puede elaborar el cuadro siguiente:

Cuadro N. ${ }^{\circ}$ 5. Producción principal de las empresas manufactureras, por estrato, según división CIIU, 2006. (Nuevos soles)

\begin{tabular}{|l|r|r|r|}
\hline \multicolumn{1}{|c|}{ CIIU } & \multicolumn{1}{c|}{ Total } & \multicolumn{1}{c|}{$\begin{array}{c}\text { De 1-9 } \\
\text { trabajadores }\end{array}$} & \multicolumn{1}{c|}{$\begin{array}{c}\text { De 10 a más } \\
\text { trabajadores }\end{array}$} \\
\hline Total & $\mathbf{1 0 1 , 6 5 5 , 0 7 9 , 5 0 7}$ & $\mathbf{1 7 , 7 9 5 , 9 7 8 , 6 1 8}$ & $\mathbf{8 3 , 8 5 9 , 1 0 0 , 8 8 9}$ \\
\hline 15 Alimentos y bebidas & $29,233,241,531$ & $5,614,087,885$ & $23,619,153,645$ \\
\hline 16 Tabaco & $2,773,673$ & 284,385 & $2,489,288$ \\
\hline 17 Productos textiles & $5,512,343,530$ & $1,208,507,403$ & $4,303,836,127$ \\
\hline 18 Prendas de vestir y art. piel & $6,471,901,482$ & $3,044,521,922$ & $3,427,379,561$ \\
\hline 19 Cuero y calzado cuero y plast. & $810,783,809$ & $422,160,490$ & $388,623,318$ \\
\hline 20 Madera y prods. madera & $1,429,263,082$ & $692,072,472$ & $737,190,611$ \\
\hline 21 Papel, libros y otros & $1,431,072,941$ & $86,914,160$ & $1,344,158,782$ \\
\hline 22 Periódicos e impresos & $7,812,425,077$ & $1,254,052,530$ & $6,558,372,547$ \\
\hline 23 Coque y refin. petróleo & $15,282,574,933$ & $7,331,927$ & $15,275,243,006$ \\
\hline 24 Químicos y derivados & $5,973,761,757$ & $425,474,929$ & $5,548,286,828$ \\
\hline 25 Caucho y plásticos & $4,035,699,766$ & $424,440,534$ & $3,611,259,231$ \\
\hline 26 Vidrio, cemento, cerámicos y ot. & $4,045,300,262$ & $420,019,562$ & $3,625,280,700$ \\
\hline 27 Hierro, acero y no ferrosos & $9,284,381,232$ & $200,609,134$ & $9,083,772,097$ \\
\hline 28 Productos metálicos & $4,463,462,185$ & $1,718,371,035$ & $2,745,091,150$ \\
\hline 29 Maquinarias, armas y otros & $980,053,110$ & $208,256,576$ & $771,796,534$ \\
\hline
\end{tabular}

(continúa...) 


\section{Gilberto José Rafael Cárdenas Núñez}

\begin{tabular}{|l|r|r|r|}
\hline 30 Otros & $86,112,385$ & $36,159,310$ & $49,953,075$ \\
\hline 31 Motores y equipos eléctricos & $1,266,565,841$ & $85,623,054$ & $1,180,942,786$ \\
\hline 32 Productos de radio y TV & $4,722,502$ & $3,700,273$ & $1,022,229$ \\
\hline 33 Equipo médico e inst. medición & $70,037,943$ & $36,848,963$ & $33,188,980$ \\
\hline 34 Automotores, partes y piezas & $400,704,897$ & $111,390,654$ & $289,314,243$ \\
\hline 35 Equipo de transporte & $352,396,102$ & $70,096,891$ & $282,299,211$ \\
\hline 36 Muebles, joyas y prod. juegos & $2,626,204,239$ & $1,674,939,263$ & $951,264,976$ \\
\hline 37 Reciclamiento met. y no met. & $79,297,227$ & $50,115,265$ & $29,181,962$ \\
\hline Fuente: Censo Nacional de Empresas Manufactureras 2007 (CENEM). \\
\hline Elaboración: PRODUCE / DGI-Dirección de Competitividad \\
\hline
\end{tabular}

El valor de ventas está relacionado con el valor neto total de la producción manufacturera. Así, las principales actividades son alimentos y bebidas, actividad que se encuentra a lo largo y ancho del país, por ser un bien de consumo habitual entre la población nacional, al igual que el consumo relativo de gaseosas o cerveza. Le sigue en orden de importancia los derivados de refinería de petróleo y acero.

\section{CONCLUSIONES}

La actividad industrial manufacturera de 10 a más trabajadores es la principal en cuanto a valor de la producción en relación con la actividad industrial manufacturera de 1 a 9 trabajadores. La relación de valores entre la primera y la segunda es de 4.88. Este indicador quiere decir que la pequeña empresa en la actividad industrial manufacturera es bastante menos importante que la empresa mediana o grande.

Las empresas industriales manufactureras tienen diferentes densidades de capital. La densidad de capital depende del número de trabajadores y del nivel de inversión. También tienen diferentes productividades, dependiendo del nivel de producción neta o valor agregado, y del número de trabajadores. En cuanto al número de trabajadores, algunas actividades industriales manufactureras son intensivas en empleo, como son las actividades agroindustriales o textiles. Otras son intensivas en capital, como el acero, cemento o petróleo.

La actividad industrial manufacturera tiene vigencia actualmente en el país. Cierto que no hay manufactura de tecnología sofisticada o intermedia, como la que se requiere 


\section{¿Existe aún la industria manufacturera en el Perú?}

en la producción de automotores o bienes de consumo duradero para uso doméstico, pero en el país existe un importante núcleo e iniciativas empresariales manufactureras, y además muchas iniciativas empresariales de pequeño alcance que significan la pequeña empresa.

El rol de la actividad manufacturera industrial está por establecerse. La carencia de planes nacionales y el abandono de los planes sectoriales, así como la reducción de los alcances del rol del Estado han afectado la configuración de la visión del país o su imagen objetivo. Muchas empresas industriales trabajan a su buen entender y posición. La articulación y potenciación de las iniciativas e inversiones de los empresarios está aún por establecerse y es necesaria por los requerimientos de mejora de la competitividad de la economía nacional.

\section{BIBLIOGRAFÍA}

Alarco, Germán; Prieto, Lorena y otros. Crisis. Análisis y perspectivas de la crisis económica mundial desde el Perú; CENTRUM; 2009, Lima.

Alvarado, Javier; Portocarrero, Felipe; Trivelli, Carolina; González, Efraín; Galarza, Francisco y Venero, Hildegardi. El financiamiento informal en el Perú; IEP ediciones, 2001, Lima.

Araoz, Mercedes; Bonifaz, José Luis; Casas, Carlos; Gonzáles Vigil, Fernando. Factores limitantes de la inversión extranjera en el Perú; Universidad del Pacífico, 2001, Lima.

Barber Kuri, Carlos Miguel; Moreno Fabre, Raúl Alejandro. Industrias representativas de América, Europa y Asia; Universidad Anáhuac, 2006, México.

Cárdenas E.; Ocampo, José Antonio; Thorp, Rosemary (compiladores). Industrialización y Estado en América Latina. Fondo de Cultura Económica, 2003, México.

Carranza Cárdenas, Octavio; El Presupuesto del Perú. Editorial Horizonte; 2006, Lima.

Castañeda Mungi, Guillermo. iFuera las caretas!; 2008, Lima. 


\section{Gilberto José Rafael Cárdenas Núñez}

Cotlear, Daniel (editor). Un nuevo contrato social para el Perú; Banco Mundial, 2006, Lima.

Dini, Marco y Stumpo, Giovanni. Pequeñas y medianas empresas y eficiencia colectiva. Estudio de caso en América Latina; CEPAL, Siglo XXI Editores; 2004, México.

Giugale, Marcelo M; Fretes-Cibils, Vicente; Newman, John L.. Perú. La oportunidad de un país diferente; 2006, Banco Mundial, Lima.

Grompone, Romeo y Tanaka, Martín. Entre el crecimiento económico y la insatisfacción social; Instituto de Estudios Peruanos; 2009, Lima.

Olmedo, Bernardo y Solleiro, José Luis. Políticas industriales y tecnológicas para las pequeñas y medianas empresas; Universidad autónoma de México, 2001, México.

Peralta, Luis. Las Pymes y las alianzas estratégicas para la exportación; Universidad de San Martín de Porres; Lima.

Pipitone, Ugo. Ciudades, naciones, regiones; Fondo de Cultura Económica; 2003, México.

Rey, Walter. Los milagros no existen para salir de la pobreza; 2007, Lima. 